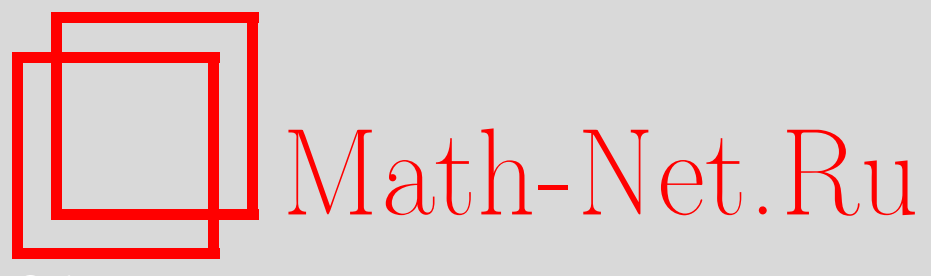

В. М. Бухштабер, О. В. Карпов, С. И. Тертычный, Математические модели динамики сильношунтированного перехода Джозефсона, УМH, 2008, том 63, выпуск $3,155-156$

DOI: https://doi.org/10.4213/rm9212

Использование Общероссийского математического портала Math-Net.Ru подразумевает, что вы прочитали и согласны с пользовательским соглашением http://www . mathnet.ru/rus/agreement

Параметры загрузки:

IP : 3.85 .5 .30

26 апреля 2023 г., 16:21:20

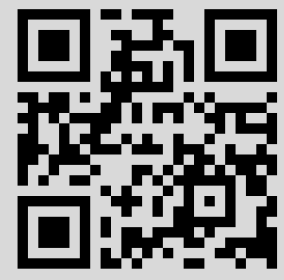




\section{Математические модели динамики сильношунтированного перехода Джозефсона}

\section{В. М. Бухштабер, О. В. Карпов, С. И. Тертычный}

Мы продолжаем начатое в [1] исследование уравнения

$$
\dot{\varphi}+\sin \varphi=q(\tau)
$$

которое при периодической функции $q(\tau)$ успешно моделирует динамику разности фаз параметра порядка в сильношунтированных переходах Джозефсона [2].

Рассмотрим общую линейную систему дифференциальных уравнений

$$
j \hbar \frac{d}{d t}\left(\begin{array}{c}
\psi_{1} \\
\psi_{2}
\end{array}\right)=\left(\begin{array}{cc}
M_{1} & j M_{2} \\
j M_{2}^{*} & -M_{1}
\end{array}\right)\left(\begin{array}{l}
\psi_{1} \\
\psi_{2}
\end{array}\right)
$$

в форме, используемой для вывода соотношений Джозефсона [3], [4], где ${ }^{*}$ - символ комплексного сопряжения, $M_{1}=M_{1}(t)$ и $M_{2}=M_{2}(t)$ - соответственно вещественная и комплексная функции вещественного аргумента $t$ и $j=\sqrt{-1}$.

Будем считать, что $M_{2}(t)$ - ненулевая периодическая функция с периодом $T_{2}$ и $\tau=$ $2 \hbar^{-1} \int_{0}^{t}\left|M_{2}\left(t^{\prime}\right)\right| d t^{\prime}$. Положим $\psi_{l}=\exp \left(\varsigma_{l}+j \chi_{l}\right)$, и $M_{2}=\left|M_{2}\right| \exp (j \delta)$, где $\varsigma_{l}=\varsigma_{l}(t)$, $\chi_{l}=\chi_{l}(t)$ и $\delta=\delta(t)$ - вещественные функции времени $t, l=1,2$. Введем новые функции $j \widetilde{\varphi}(\tau)=\left[\hat{\varsigma}_{2}(\tau)-\hat{\varsigma}_{1}(\tau)\right]+j\left[\hat{\chi}_{2}(\tau)-\hat{\chi}_{1}(\tau)\right]+j \hat{\delta}(\tau)$ и $\tilde{\xi}(\tau)=\left[\hat{\varsigma}_{2}(\tau)+\hat{\varsigma}_{1}(\tau)\right]+$ $j\left[\hat{\chi}_{2}(\tau)+\widehat{\chi}_{1}(\tau)\right]$, где $\hat{\varsigma}_{l}(\tau)=\varsigma_{l}(t), \hat{\chi}_{l}(\tau)=\chi_{l}(t)$ и $\hat{\delta}(\tau)=\delta(t)$. Заметим, что из вида матрицы системы (2) следует закон сохранения: $\left|\psi_{2}\right|^{2}-\left|\psi_{1}\right|^{2}=\rho_{0}=$ const.

Лемма 1. Система уравнений (2) эквивалентна двум нелинейным уравнениям $\dot{\tilde{\varphi}}+\sin \widetilde{\varphi}=q(\tau) u \dot{\tilde{\xi}}-\cos \widetilde{\varphi}=0$, где $q(\tau)=M_{1}\left|M_{2}\right|^{-1}+\hat{\delta}(\tau)$.

Положим $\widetilde{\varphi}(\tau)=\varphi(\tau)-j \varkappa(\tau)$ и $\tilde{\xi}(\tau)=\xi(\tau)-j \phi(\tau)$, где $\varphi(\tau), \varkappa(\tau), \xi(\tau)$ и $\phi(\tau)-$ вещественные функции времени $\tau$. Тогда два уравнения из леммы 1 преобразуются в следующие четыре уравнения:

$$
\dot{\varphi}+\operatorname{ch} \varkappa \sin \varphi=q(\tau), \quad \dot{\varkappa}+\operatorname{sh} \varkappa \cos \varphi=0, \quad \dot{\xi}-\operatorname{ch} \varkappa \cos \varphi=0, \quad \dot{\phi}+\operatorname{sh} \varkappa \sin \varphi=0 .
$$

Теорема 1. Система (2) эквивалентна уравнению (1) с $q(\tau)=M_{1}\left|M_{2}\right|^{-1}+\dot{\hat{\delta}}(\tau)$ тогда и толъко тогда, когда $\rho_{0}=0,\left|M_{2}\right|=$ const $u \dot{\xi}=\cos \varphi$.

Снимем условия $\rho_{0}=0$ и $\left|M_{2}\right|=$ const.

Теорема 2. Пусть $q(\tau)$ - периодическая функиия с периодом Т. Решение системы уравнений из леммы 1 поточечно сходится $к$ решению уравнения (1), когда существует и не равен нулю предел $\lambda=\frac{1}{2} \lim _{n \rightarrow \pm \infty}\left[T^{-1} \int_{\tau-T / 2}^{\tau+T / 2} \cos \varphi\left(\tau^{\prime}+n T\right) d \tau^{\prime}\right]$. ЛЕмма 2. Функиия $W(\tau)=W_{0} \exp S(\tau)$, где $2 S(\tau)=\int_{\tau_{0}}^{\tau}\left[\cos \varphi\left(\tau^{\prime}\right)-j \sin \varphi\left(\tau^{\prime}\right)\right] d \tau^{\prime}$
является решением уравнения

$$
\ddot{W}(\tau)+j q(\tau) \dot{W}(\tau)-\frac{1}{4} W(\tau)=0
$$

тогда и только тогда, когда функция $\varphi(\tau)$ является решением уравнения (1).

Работа выполнена при поддержке РФФИ (гранты № 06-01-00486-a, 07-01-12064-офи). 
Пусть $q(\tau)=b+a \cos (\mu \tau)$, где $a, b$ и $\mu$ - вещественные константы. Пусть $\lambda$ из теоремы 2 существует и имеет ненулевое значение. Так как $\langle\cos \varphi(\tau)\rangle=2 \lambda$ и $\langle\sin \varphi(\tau)\rangle=b-m \mu$ [2] (угловые скобки обозначают операцию усреднения по времени, использованную в теореме 2$)$, то $W(\tau, m)=R(\tau) \exp (\Lambda \tau)$, где $2 \Lambda=2 \lambda+j(m \mu-b)$, $m$ - номер ступеньки Шапиро [2] и

$$
R(\tau)=W_{0}(m) \exp \left\{\frac{1}{2} \int_{\tau_{0}}^{\tau}\left\{\left[\cos \varphi\left(\tau^{\prime}\right)-\left\langle\cos \varphi\left(\tau^{\prime}\right)\right\rangle\right]-j\left[\sin \varphi\left(\tau^{\prime}\right)-\left\langle\sin \varphi\left(\tau^{\prime}\right)\right\rangle\right]\right\} d \tau^{\prime}\right\} .
$$

Перейдем из области $\mathbb{C} \backslash\{0\}$ на риманову поверхность функции $\ln z$ и произведем в (3) замену $\tau \rightarrow z=\exp (j \mu \tau)$ и $W(\tau) \rightarrow V(z)$. Аналитически продолжим функцию $q(\tau)$ на плоскость комплексной переменной $z$ по формуле $\tilde{q}(z)=b+(a / 2)\left(z+z^{-1}\right)$. После преобразований получим уравнение на функцию $V(z)=V$ в виде

$$
\mathbf{D}^{2} V+\left[\gamma\left(z+z^{-1}\right)+c\right] \mathbf{D} V+\vartheta V=0
$$

где $\mathbf{D}=z d / d z, \gamma=a /(2 \mu), c=b / \mu$ и $\vartheta=(2 \mu)^{-2}$. Заметим, что уравнение (5) эквивалентно дважды конфлюентному уравнению Гойна [5].

Решение уравнения (5) при условии захвата фазы ищем в виде $V(z)=z^{\eta} e^{-\gamma z} E(z)$, где $\eta$ - акцессорный комплекснозначный параметр, $E(z)$ - аналитическая по $z$ функция, удовлетворяющая уравнению $\mathbf{L} E=0 \mathbf{c} \mathbf{L}=\gamma(\mathbf{D}+\eta)+z\left\{\mathbf{D}^{2}+(2 \eta-c) \mathbf{D}+\right.$ $\left.\left[\eta^{2}-\gamma^{2}-\eta(c+1)+\vartheta\right]\right\}-\gamma z^{2}(\mathbf{D}-c+\eta)$. Положим $E(z)=E_{+}(z)+E_{-}(z)-A_{0}$, где $E_{ \pm}(z)=\sum_{k=0}^{\infty} A_{ \pm k} z^{ \pm k}$.

Лемма 3. Коэффициенты $A_{ \pm k}$ в рядах $E_{ \pm}(z)$ выражаются через сходящиеся бесконечные произведения явно заданных $(2 \times 2)$-матриц.

Введем вспомогательную функцию $Q(\eta)=\gamma(1+\eta) A_{1}+\left[\eta^{2}-\gamma^{2}-\eta(c+1)+\vartheta\right] A_{0}+$ $\gamma(c-2 \eta) A_{-1}$, тогда уравнение $\mathbf{L} E=0$ становится эквивалентным условию $Q(\eta)=0$.

Теорема 3. Пусть $\eta=-[(b+\mu(1-2 \varepsilon)) /(2 \mu)+j v]$ есть решение уравнения $Q(\eta)=0$. Тогда если $v \neq 0$, то параметрь $a, b$ и $\mu$ функиии $q(\tau)$ лежат внутри областей захвата фазы с порлдковым номером $m$ (см. [1], [2]). В этом случае $v=2 \lambda / \mu$, а номер $m$ вычисляется как

$$
m=\varepsilon+\frac{1}{\pi j} \oint \frac{E^{\prime}(z)}{E(z)} d z, \quad \varepsilon=\left\{\begin{aligned}
0 & \text { для } m \text { четных }, \\
-1 & \text { для } m \text { нечетных. }
\end{aligned}\right.
$$

Следствие 1. Решение уравнения (5) при условии захвата фазы может быть представлено в виде $V(z)=z^{-j(\Lambda / \mu)} e^{-\gamma z} \widetilde{E}(z)$, где функция $\widetilde{E}(z)$ переходит в $R(\tau)$ (см. (4)) при замене z нат и $\oint\left[\widetilde{E}^{\prime}(z) / \widetilde{E}(z)\right] d z=0$.

\section{Список литературы}

[1] В. М. Бухштабер, О.В. Карпов, С. И. Тертычный, УМН, 59:2 (2004), 187-188. [2] В. М. Бухштабер, О.В. Карпов, С.И. Тертычный, ЖЭТФ, 124:3(9) (2003), 691-699. [3] К. К. Лихарев, Б. Е. Ульрих, Системы с джозефсоновскими контактами, МГУ, М., 1978. [4] А. Бароне, Дж. Патерно, Эффект Джозефсона. Физика и применение, Мир, М., 1984. [5] С. Славянов, В. Лай, Специальные функиии: единая теория, основанная на анализе особенностей, Невский диалект, С.-Петербург, 2002.

B. M. Бухштабер (V. M. Buchstaber)

Математический институт им. В. А. Стеклова РАН

E-mail: buchstab@mi.ras.ru

О. В. Карпов (O. V. Karpov)

ВНИИФТРИ

E-mail: mera@vniiftri.org

C. И. Тертычный (S. I. Tertychnyi)

ВНИИФТРИ
Представлено С. П. Новиковым Принято редколлегией 06.05.2008 